Research Article

\title{
Double Controlled Dislocated Quasi-Metric Type Spaces and Some Results
}

\author{
Abdullah Shoaib $\mathbb{D}^{1},{ }^{1}$ Shaif S. Alshoraify, ${ }^{2}$ and Muhammad Arshad $\mathbb{D D}^{2}$ \\ ${ }^{1}$ Department of Mathematics and Statistics, Riphah International University, Islamabad, Pakistan \\ ${ }^{2}$ Department of Mathematics and Statistics, International Islamic University, H-10, Islamabad 44000, Pakistan \\ Correspondence should be addressed to Abdullah Shoaib; abdullahshoaib15@yahoo.com
}

Received 20 March 2020; Accepted 15 May 2020; Published 8 July 2020

Academic Editor: Tepper L. Gill

Copyright (C) 2020 Abdullah Shoaib et al. This is an open access article distributed under the Creative Commons Attribution License, which permits unrestricted use, distribution, and reproduction in any medium, provided the original work is properly cited.

\begin{abstract}
The aim of this work is to introduce double controlled dislocated quasi-metric type spaces and to obtain fixed point results for a pair of multivalued mappings satisfying Kannan-type double controlled contraction in such spaces. An example has been built and a remark has been given which shows that how our result can be used when a corresponding new result in dislocated quasi $b$-metric type spaces cannot be used. Our results generalize and extend many existing results in the literature.
\end{abstract}

\section{Introduction and Preliminaries}

The field of fixed point theory covers both pure and applied mathematics. Fixed point theory is a special branch of functional analysis and its results are used to show the solution existence of different mathematical models. A multivalued mapping $B$ from $C$ to the subsets of $C$ has a fixed point $y \in C$, if $y \in B y$. If we take elements of $C$ instead of subsets of $C$, then $B$ represents a single valued mapping from $C$ to $C$. A single valued mapping $B: C \longrightarrow C$ has a fixed point $y \in C$ if $y=B y$. Fixed point results for multivalued mappings generalize the results for single-valued mappings. Fixed point results of multivalued mappings have applications in engineering, economics, Nash equilibria, and game theory [1-4]. Due to its importance, many interesting results have been proved in the setting of multivalued mappings, for example, see [5-14].

By excluding one and a half condition, out of three conditions of a metric space, we obtain dislocated quasimetric space [11]. Complete dislocated quasi-metric space is a generalization of 0 -complete and complete quasi-partial metric space [15-17]. Dislocated quasi-metric also generalizes dislocated metric, partial metric, and quasi-metric. Fixed point results in dislocated quasi-metric space can be seen in [18-22].
Ran and Reurings [23] gave a fixed point result with an order and obtained solution to matrix equations as an application. Nieto et al. [24] gave an extension to the result in [23] for ordered mappings and used it to give a unique solution for ODE with periodic boundary conditions. Altun et al. [25] introduced a new approach to common fixed point of mappings, satisfying a generalized contraction with a new restriction in a complete ordered metric space.

On the contrary, Kamran et al. [26] introduced a new concept of generalized $b$ metric space, named as extended b-metric spaces, see also [27]. They replaced the parameter $b \geq 1$ in triangle inequality by the control function $\theta: X \times X \longrightarrow[1, \infty)$. Recently, Mlaiki et al. [27] generalized the triangle inequality in $b$-metric space by using control function in a different style and introduced controlled metric type spaces. Very recently, Abdeljawad et al. [28] generalized the concept of controlled metric type space by introducing two control functions $\alpha(r, y)$ and $\mu(r, y)$ and establishing double controlled metric type space. In this paper, we extend the result of Altun et al. [25] in four different ways by using

(i) Multivalued mappings instead of single-valued mappings

(ii) Kannan-type contraction instead of Banach-type contraction 
(iii) Left $K$-sequentially complete double controlled dislocated quasi-metric type space instead of complete metric space

(iv) A function which generalized the partial order relation

Our results unify, extend, and generalize several comparable results in the existing literature. We give the following definitions and results which will be useful to understand the paper.

Definition 1 (see [28]). Given noncomparable functions $\alpha, \mu: \mathfrak{J} \times \mathfrak{J} \longrightarrow[1, \infty)$. Suppose that $q: \mathfrak{I} \times \mathfrak{J} \longrightarrow[0, \infty)$ satisfies the following:

(q1) $q(r, y)=0$ if and only if $r=y$

(q2) $q(r, y)=q(y, r)$

(q3) $q(r, y) \leq \alpha(r, z) q(r, z)+\mu(z, y) q(z, y)$, for all $r, z, y \in \mathfrak{J}$

Then, $q$ is called double controlled metric type with the functions $\alpha$ and $\mu$, and the pair $(\mathfrak{I}, q)$ is called double controlled metric type space with the functions $\alpha$ and $\mu$.

Theorem 1 (see [28]). Let $(\mathfrak{I}, q)$ be a complete double controlled metric type space with the functions $\alpha, \mu: \mathfrak{I} \times \mathfrak{I} \longrightarrow[1, \infty)$ and let $T: \mathfrak{I} \longrightarrow \mathfrak{I}$ be a given mapping. Suppose that the following conditions are satisfied.

There exists $k \in(0,1)$ such that

$$
q(T(x), T(y)) \leq k q(x, y), \quad \text { for all } x, y \in \mathfrak{I} .
$$

For $v_{0} \in \mathfrak{J}$, choose $v_{n}=T^{n} v_{0}$. Assume that

$$
\sup _{m \geq 1} \lim _{i \longrightarrow \infty} \frac{\alpha\left(v_{i+1}, v_{i+2}\right)}{\alpha\left(v_{i}, v_{i+1}\right)} \mu\left(v_{i+1}, v_{m}\right)<\frac{1}{k} .
$$

In addition, for every $v \in \mathfrak{J}$, we have

$$
\lim _{i \longrightarrow \infty} \alpha\left(v, v_{n}\right) \lim _{i \longrightarrow \infty} \mu\left(v_{n}, v\right) \text { exist and are finite. }
$$

Then, $T$ has a unique fixed point $v^{*} \in \mathfrak{\Im}$.

Definition 2. Given noncomparable functions $\alpha, \mu: \mathfrak{I} \times \mathfrak{I} \longrightarrow[1, \infty)$. If $\rho_{q}: \mathfrak{I} \times \mathfrak{I} \longrightarrow[0, \infty)$ satisfies

$$
\begin{aligned}
& \left(\rho_{q 1}\right) q(\omega, v)=\rho_{q}(v, \omega)=0, \quad \text { then } \omega=v, \\
& \left(\rho_{q 2}\right) q(\omega, v) \leq \alpha(\omega, e) q(\omega, e)+\mu(e, v) q(e, v),
\end{aligned}
$$

for all $\omega, v, e \in \mathfrak{I}$, then $\rho_{q}$ is called a double controlled dislocated quasi-metric type with the functions $\alpha$ and $\mu$ and $\left(\mathfrak{I}, \rho_{q}\right)$ is called a double controlled dislocated quasi-metric type space. If $\mu(e, v)=\alpha(e, v)$ then $\left(\mathfrak{J}, \rho_{q}\right)$ is called a controlled quasi-metric type space.

Remark 1. Any dislocated quasi-metric space or double controlled metric-type space is double controlled dislocated quasi-metric type space, but the converse is not true in general. Also, a controlled dislocated quasi-metric type space is also double controlled quasi-metric type space. The converse is not true in general (see Examples 1 and 2).

Example 1. Let $\mathfrak{J}=\{0,1,2,3\}$. Define $\rho_{q}: \mathfrak{I} \times \mathfrak{I} \longrightarrow[0, \infty)$ by $\quad \rho_{q}(0,1)=0, \quad \rho_{q}(0,2)=1, \quad \rho_{q}(0,3)=(1 / 4)$, $\rho_{q}(1,0)=(1 / 2), \rho_{q}(1,2)=2, \quad \rho_{q}(1,3)=(1 / 3), \rho_{q}(2,0)=$ $(1 / 2), \quad \rho_{q}(2,1)=1, \quad \rho_{q}(2,3)=(1 / 3), \rho_{q}(3,0)=(3 / 2)$, $\rho_{q}(3,1)=2, \rho_{q}(3,2)=(1 / 4), \rho_{q}(0,0)=(1 / 2), \quad \rho_{q}(1,1)=0$, $\rho_{q}(2,2)=2$, and $\rho_{q}(3,3)=0$.

$$
\text { Define } \quad \alpha, \mu: \mathfrak{I} \times \mathfrak{I} \longrightarrow[1, \infty) \text { as }
$$

$\alpha(0,1)=\alpha(1,2)=\alpha(0,2)=1, \alpha(1,0)=(4 / 3), \alpha(2,0)=2$, $\alpha(3,1)=(4 / 3), \quad \alpha(2,3)=3, \quad \alpha(0,3)=(4 / 3), \quad \alpha(1,3)=$ $3, \alpha(2,1)=1, \quad \alpha(3,0)=(4 / 3), \alpha(3,2)=2, \alpha(0,0)=\alpha(1,1)$ $=\alpha(2,2)=\alpha(3,3)=1$,

$$
\mu(1,2)=\mu(2,1)=(3 / 2), \mu(2,0)=2, \mu(3,0)=\mu(0,3)=
$$
$\mu(1,0)=\mu(0,1)=\mu(1,3)=\mu(3,1)=1, \mu(3,2)=4, \mu(2,3)$ $=1, \mu(0,2)=(4 / 3)$, and $\mu(0,0)=\mu(1,1)=\mu(2,2)=\mu$ $(3,3)=1$. It is obvious that $\rho_{q}$ is double controlled dislocated quasi-metric type for all $\omega, v, e \in \mathfrak{J}$. It is clear that $\rho_{q}$ is not double controlled metric-type space. Also, it is not controlled dislocated quasi-metric type. Indeed,

$$
\rho_{q}(1,2)=2>\frac{3}{2}=\alpha(1,3) \rho_{q}(1,3)+\alpha(3,2) \rho_{q}(3,2) .
$$

Definition 3. Let $\left(\mathfrak{I}, \rho_{q}\right)$ be a double controlled dislocated quasi-metric type space:

(i) A sequence $\left\{r_{n}\right\}$ in $\left(\mathfrak{\Im}, \rho_{q}\right)$ is called left $K$-Cauchy if $\forall \varepsilon>0, \quad \exists n_{0} \in N$ such that $\rho_{q}\left(r_{m}, r_{n}\right)<\varepsilon$ and $\forall n>m>n_{0}$.

(ii) A sequence $\left\{r_{n}\right\}$ is double controlled dislocated quasi and converges to $r$ if $\lim _{n \longrightarrow \infty} \rho_{q}\left(r_{n}, r\right)=\lim _{n \longrightarrow \infty} \rho_{q}\left(r, r_{n}\right)=0$ or for any $\varepsilon>0$, there exists $n_{0} \in N$, such that, for all $n>n_{0}, \rho_{q}\left(r, r_{n}\right)<\varepsilon$ and $\rho_{q}\left(r_{n}, r\right)<\varepsilon$. In this case, $r$ is called a $\rho_{q}$-limit of $\left\{r_{n}\right\}$.

(iii) $\left(\mathfrak{J}, \rho_{q}\right)$ is called left $K$-sequentially complete if every left $K$-Cauchy sequence in $\left(\mathfrak{I}, \rho_{q}\right)$ converges to a point $r \in \mathfrak{I}$ such that $\rho_{q}(r, r)=0$.

Definition 4. Let $\left(\mathfrak{I}, \rho_{q}\right)$ be a double controlled dislocated quasi-metric type space. Let $K$ be a nonempty subset of $\mathfrak{I}$ and let $r \in \mathfrak{J}$. An element $y_{0} \in K$ is called a best approximation in $K$ if

$$
\begin{aligned}
& \rho_{q}(r, K)=\rho_{q}\left(r, y_{0}\right), \quad \text { where } \rho_{q}(r, K)=\inf _{y \in K} \rho_{q}(r, y), \\
& \rho_{q}(K, r)=\rho_{q}\left(y_{0}, r\right), \quad \text { where } \rho_{q}(K, r)=\inf _{y \in K} \rho_{q}(y, r) .
\end{aligned}
$$

If each $r \in \mathfrak{J}$ has at least one best approximation in $K$, then $K$ is called a proximinal set. We denote the set of all proximinal subsets of $\mathfrak{I}$ by $P(\mathfrak{I})$.

Definition 5 (see [11]). The function $H_{\rho_{q}}: P(\mathfrak{I}) \times P(\mathfrak{I}) \longrightarrow[0, \infty)$, defined by 


$$
H_{\rho_{q}}(A, B)=\max \left\{\sup _{a \in A} \rho_{q}(a, B), \sup _{b \in B} \rho_{q}(A, b)\right\},
$$

is called double controlled dislocated quasi-Hausdorff metric type on $P(\mathfrak{I})$. Also, $\left(P(\Im), H_{\rho_{q}}\right)$ is known as double controlled dislocated quasi-Hausdorff metric type space.

Following similar arguments of Lemma 1.7 given by Shoaib [11], we obtain the following lemma.

Lemma 1. Let $\left(\Im, \rho_{q}\right)$ be a double controlled dislocated quasi-metric type space. Let $\left(P(\Im), H_{\rho_{q}}\right)$ be a double controlled dislocated quasi-Hausdorff metric type space on $P(\mathfrak{I})$. Then, for all $A, B \in P(\Im)$ and for each $a \in A$, there exists $b_{a} \in B$, such that $H_{\rho_{q}}(A, B) \geq \rho_{q}\left(a, b_{a}\right)$ and $H_{\rho_{q}}(B, A) \geq$ $\rho_{q}\left(b_{a}, a\right)$.

\section{Main Result}

Let $\left(\mathfrak{\Im}, \rho_{q}\right)$ be a double controlled complete dislocated quasimetric type space and $r_{0} \in \mathfrak{I}$ and $S, T: \mathfrak{I} \longrightarrow P(\mathfrak{I})$ be multifunctions on $\mathfrak{I}$. Let $r_{1} \in S r_{0}$ be an element such that $\rho_{q}\left(r_{0}, S r_{0}\right)=\rho_{q}\left(r_{0}, r_{1}\right)$ and $\rho_{q}\left(S r_{0}, r_{0}\right)=\rho_{q}\left(r_{1}, r_{0}\right)$. Let $r_{2} \in \operatorname{Tr}_{1}$ be such that $\rho_{q}\left(r_{1}, T r_{1}\right)=\rho_{q}\left(r_{1}, r_{2}\right)$ and $\rho_{q}\left(\operatorname{Tr}_{1}, r_{1}\right)=\rho_{q}\left(r_{2}, r_{1}\right)$. Let $r_{3} \in S r_{2}$ be such that $\rho_{q}\left(r_{2}, S r_{2}\right)=\rho_{q}\left(r_{2}, r_{3}\right)$ and so on. Thus, we construct a sequence $r_{n}$ of points in $\mathfrak{J}$ such that $r_{2 n+1} \in S r_{2 n}$ and $r_{2 n+2} \in T r_{2 n+1}$, with $\quad \rho_{q}\left(r_{2 n}, S r_{2 n}\right)=\rho_{q}\left(r_{2 n}, r_{2 n+1}\right)$, $\rho_{q}\left(S r_{2 n}, r_{2 n}\right)=\rho_{q}\left(r_{2 n+1}, r_{2 n}\right), \quad \rho_{q}\left(r_{2 n+1}, T r_{2 n+1}\right)=$ $\rho_{q}\left(r_{2 n+1}, r_{2 n+2}\right)$, and $\rho_{q}\left(\operatorname{Tr}_{2 n+1}, r_{2 n+1}\right)=\rho_{q}\left(r_{2 n+2}, r_{2 n+1}\right)$, where $n=0,1,2, \ldots$. We denote this iterative sequence by $\left\{T S\left(r_{n}\right)\right\}$. We say that $\left\{T S\left(r_{n}\right)\right\}$ is a sequence in $\mathfrak{I}$ generated by $r_{0}$. If $T=S$, then we say that $\left\{T\left(r_{n}\right)\right\}$ is a sequence in $\mathfrak{J}$ generated by $r_{0}$. Let $M \subseteq \mathfrak{I}$, define $\beta^{*}(r, M)=\inf \{\beta(r, a), a \in M\}$ and $\beta^{*}(M, y)=\inf \{\beta(b, y)$, $b \in M\}$.

Definition 6. Let $\mathfrak{I}$ be a nonempty set, $\beta: \mathfrak{I} \times \mathfrak{I} \longrightarrow[0,+\infty)$ be a mapping, and $S, T: \mathfrak{I} \longrightarrow P(\mathfrak{I})$ be the multivalued mappings; then, the pair $(S, T)$ is said to be a pair of $\beta^{*}$-Alt multivalued mapping, if

$$
\begin{aligned}
\text { (a) } \beta^{*}(r, S r) & \geq 1, \rho_{q}(r, S r)=\rho_{q}(r, y), \\
\rho_{q}(S r, r) & =\rho_{q}(y, r) \text { imply } \beta^{*}(S y, y) \geq 1, \\
\text { (b) } \beta^{*}(S r, r) & \geq 1, \operatorname{rho}_{q}(r, T r)=\rho_{q}(r, y), \\
\rho_{q}(\operatorname{Tr}, r) & =\rho_{q}(y, r) \text { imply } \beta^{*}(y, S y) \geq 1 .
\end{aligned}
$$

Definition 7. Let $\left(\mathfrak{I}, \rho_{q}\right)$ be a complete double controlled dislocated quasi-metric type space and $(S, T)$ be a pair of $\beta^{*}$-Alt multivalued mapping. Then, $(S, T)$ is called $\beta^{*}$ Kannan-type double controlled contraction, if for every two consecutive points $x, y$ belonging to the range of an iterative sequence $\left\{T S\left(r_{n}\right)\right\}$ with $\beta^{*}(S y, y) \geq 1$ and $\beta^{*}(x, S x) \geq 1$, and we have

$$
\max \left\{H_{\rho_{q}}(S x, T y), H_{\rho_{q}}(T y, S x)\right\} \leq t\left(\rho_{q}(y, T y)+\rho_{q}(x, S x)\right),
$$

where $t \in[0,(1 / 2))$. Also, the terms of the sequence $\left\{T S\left(r_{n}\right)\right\}$ satisfy the following:

$$
\sup _{m \geq 1} \lim _{i \longrightarrow \infty} \frac{\alpha\left(r_{i+1}, r_{i+2}\right)}{\alpha\left(r_{i}, r_{i+1}\right)} \mu\left(r_{i+1}, r_{m}\right)<\frac{1-t}{t} .
$$

Theorem 2. Let $\left(\Im, \rho_{q}\right)$ be a left K-sequentially complete double controlled dislocated quasi-metric type space. $(S, T)$ be the pair of $\beta^{*}$ Kannan-type double controlled contraction. Assume that

(i) The set $G(S)=\left\{r: \beta^{*}(r, S r) \geq 1\right\}$ contains $r_{0}$ and is closed.

(ii) For every $r \in \mathfrak{I}$, we have

$$
\lim _{n \longrightarrow \infty} \alpha\left(r, r_{n}\right) \lim _{n \longrightarrow \infty} \mu\left(r_{n}, r\right)<\frac{1}{t} .
$$

Then, $\left\{T S\left(r_{n}\right)\right\} \longrightarrow r \in \mathfrak{J}$. Also, if (9) holds for each $r, y \in\left\{r^{*}\right\}$, then $S$ and $T$ have a common fixed point $r^{*}$ in $\mathfrak{J}$ and $\rho_{q}\left(r^{*}, r^{*}\right)=0$.

Proof. As $r_{0}$ be an arbitrary element of $G(S)$, from condition (i) $\beta^{*}\left(r_{0}, S r_{0}\right) \geq 1$. Let $\left\{T S\left(r_{n}\right)\right\}$ be the iterative sequence in $\mathfrak{I}$ generated by a point $r_{0} \in \mathfrak{I}$. Since $\beta^{*}\left(r_{0}, S r_{0}\right) \geq 1$, $\rho_{q}\left(r_{0}, S r_{0}\right)=\rho_{q}\left(r_{0}, r_{1}\right)$ and $\rho_{q}\left(S r_{0}, r_{0}\right)=\rho_{q}\left(r_{1}, r_{0}\right)$. As $(S, T)$ is $\beta^{*}$-Alt multivalued mapping, so $\beta^{*}\left(S r_{1}, r_{1}\right) \geq 1$. Now, $\beta^{*}\left(S_{1}, r_{1}\right) \geq 1, \quad \rho_{q}\left(r_{1}, \operatorname{Tr}_{1}\right)=\rho_{q}\left(r_{1}, r_{2}\right), \quad$ and $\rho_{q}\left(\operatorname{Tr}_{1}, r_{1}\right)=\rho_{q}\left(r_{2}, r_{1}\right)$ imply that $\beta^{*}\left(r_{2}, S r_{2}\right) \geq 1$. By induction, we deduce that $\beta^{*}\left(r_{2 p}, S r_{2 p}\right) \geq 1$ and $\beta^{*}\left(S r_{2 p+1}, r_{2 p+1}\right) \geq 1$, for all $p=0,1,2, \cdots$. Now, by Lemma 1 , we have

$$
\begin{gathered}
\rho_{q}\left(r_{2 p}, r_{2 p+1}\right) \leq H_{\rho_{q}}\left(T r_{2 p-1}, S r_{2 p}\right), \\
\rho_{q}\left(r_{2 p+1}, r_{2 p+2}\right) \leq H_{\rho_{q}}\left(S r_{2 p}, T r_{2 p+1}\right) .
\end{gathered}
$$

As $\quad r_{2 p}, r_{2 p+1} \in\left\{T S\left(r_{n}\right)\right\}, \quad \beta^{*}\left(r_{2 p}, S r_{2 p}\right) \geq 1, \quad$ and $\beta^{*}\left(S r_{2 p+1}, r_{2 p+1}\right) \geq 1$, then by using condition (9) in inequality (13), we have

$$
\begin{aligned}
\rho_{q}\left(r_{2 p+1}, r_{2 p+2}\right) & \leq t\left[\rho_{q}\left(r_{2 p}, S r_{2 p}\right)+\rho_{q}\left(r_{2 p+1}, T r_{2 p+1}\right)\right] \\
& \leq t\left(\rho_{q}\left(r_{2 p}, r_{2 p+1}\right)+\rho_{q}\left(r_{2 p+1}, r_{2 p+2}\right)\right) \\
& \leq \frac{t}{1-t}\left(\rho_{q}\left(r_{2 p}, r_{2 p+1}\right)\right), \\
\rho_{q}\left(r_{2 p+1}, r_{2 p+2}\right) & \leq \dot{c}\left(\rho_{q}\left(r_{2 p}, r_{2 p+1}\right)\right), \quad \text { where } \dot{c}=\frac{t}{1-t} .
\end{aligned}
$$


As $\quad r_{2 p}, r_{2 p-1} \in\left\{T S\left(r_{n}\right)\right\}, \quad \beta^{*}\left(r_{2 p}, S r_{2 p}\right) \geq 1, \quad$ and $\beta^{*}\left(S r_{2 p-1}, r_{2 p-1}\right) \geq 1$, then by using condition (9) in inequality (12), we obtain

$$
\begin{aligned}
\rho_{q}\left(r_{2 p}, r_{2 p+1}\right) & \leq t\left(\rho_{q}\left(r_{2 p-1}, T r_{2 p-1}\right)+\rho_{q}\left(r_{2 p}, S r_{2 p}\right)\right) \\
& \leq t\left(\rho_{q}\left(r_{2 p-1}, r_{2 p}\right)+\rho_{q}\left(r_{2 p}, r_{2 p+1}\right)\right) \\
& \leq \frac{t}{1-t}\left(\rho_{q}\left(r_{2 p-1}, r_{2 p}\right)\right), \\
\rho_{q}\left(r_{2 p}, r_{2 p+1}\right) & \leq \dot{c}\left(\rho_{q}\left(r_{2 p-1}, r_{2 p}\right)\right) .
\end{aligned}
$$

As $r_{2 p-2}, r_{2 p-1} \in\left\{T S\left(r_{n}\right)\right\}, \quad \beta^{*}\left(r_{2 p-2}, S r_{2 p-2}\right) \geq 1$, and $\beta^{*}\left(S r_{2 p-1}, r_{2 p-1}\right) \geq 1$, then by using condition (9), we obtain $\rho_{q}\left(r_{2 p-1}, r_{2 p}\right) \leq t\left(\rho_{q}\left(r_{2 p-2}, S r_{2 p-2}\right)+\rho_{q}\left(r_{2 p-1}, T r_{2 p-1}\right)\right)$

$$
\begin{aligned}
& \leq t\left(\rho_{q}\left(r_{2 p-2}, r_{2 p-1}\right)+\rho_{q}\left(r_{2 p-1}, r_{2 p}\right)\right) \\
& \leq \frac{t}{1-t}\left(\rho_{q}\left(r_{2 p-2}, r_{2 p-1}\right)\right) \leq \dot{c}\left(\rho_{q}\left(r_{2 p-2}, r_{2 p-1}\right)\right) .
\end{aligned}
$$

Using (16) in (15), we have

$$
\rho_{q}\left(r_{2 p}, r_{2 p+1}\right) \leq \dot{c}^{2} \rho_{q}\left(r_{2 p-2}, r_{2 p-1}\right) \text {. }
$$

As $r_{2 p-2}, r_{2 p-1} \in\left\{T S\left(r_{n}\right)\right\}, \quad \beta^{*}\left(S r_{2 p-1}, r_{2 p-1}\right) \geq 1$, and $\beta^{*}\left(r_{2 p-2}, S r_{2 p-2}\right) \geq 1$, then by using condition (9), we obtain

$$
\begin{aligned}
\rho_{q}\left(r_{2 p-2}, r_{2 p-1}\right) & \leq t\left(\rho_{q}\left(r_{2 p-3}, r_{2 p-2}\right)+\rho_{q}\left(r_{2 p-2}, r_{2 p-1}\right)\right) \\
& \leq \dot{c}^{3}\left(\rho_{q}\left(r_{2 p-3}, r_{2 p-2}\right)\right) .
\end{aligned}
$$

From (17) and (18), we have

$$
\dot{c}^{2}\left(\rho_{q}\left(r_{2 p-2}, r_{2 p-1}\right)\right) \leq \dot{c}^{3}\left(\rho_{q}\left(r_{2 p-3}, r_{2 p-2}\right)\right) .
$$

Using (19) in (15), we have

$$
\rho_{q}\left(r_{2 p}, r_{2 p+1}\right) \leq \dot{c}^{3}\left(\rho_{q}\left(r_{2 p-3}, r_{2 p-2}\right)\right) .
$$

Continuing in this way, we obtain

$$
\rho_{q}\left(r_{2 p}, r_{2 p+1}\right) \leq \dot{c}^{2 \mathrm{p}}\left(\rho_{q}\left(r_{0}, r_{1}\right)\right) .
$$

Similarly, by using (14)-(16) and continuing in this way, we obtain

$$
\rho_{q}\left(r_{2 p+1}, r_{2 p+2}\right) \leq \dot{c}^{2 p+1}\left(\rho_{q}\left(r_{0}, r_{1}\right)\right) .
$$

Combining inequalities (21) and (22), we have

$$
\rho_{q}\left(r_{n}, r_{n+1}\right) \leq \mathrm{c}^{\mathrm{n}}\left(\rho_{q}\left(r_{0}, r_{1}\right)\right) .
$$

Now, to prove that $\left\{r_{n}\right\}$ is a left Cauchy sequence, for all natural numbers $n<m$, we have

$$
\begin{aligned}
\rho_{q}\left(r_{n}, r_{m}\right) \leq & \alpha\left(r_{n}, r_{n+1}\right) \rho_{q}\left(r_{n}, r_{n+1}\right)+\mu\left(r_{n+1}, r_{m}\right) \rho_{q}\left(r_{n+1}, r_{m}\right) \\
\leq & \alpha\left(r_{n}, r_{n+1}\right) \rho_{q}\left(r_{n}, r_{n+1}\right)+\mu\left(r_{n+1}, r_{m}\right) \alpha\left(r_{n+1}, r_{n+2}\right) \rho_{q}\left(r_{n+1}, r_{n+2}\right)+\mu\left(r_{n+1}, r_{m}\right) \mu\left(r_{n+2}, r_{m}\right) \rho_{q}\left(r_{n+2}, r_{m}\right) \\
\leq & \alpha\left(r_{n}, r_{n+1}\right) \rho_{q}\left(r_{n}, r_{n+1}\right)+\mu\left(r_{n+1}, r_{m}\right) \alpha\left(r_{n+1}, r_{n+2}\right) \rho_{q}\left(r_{n+1}, r_{n+2}\right)+\mu\left(r_{n+1}, r_{m}\right) \mu\left(r_{n+2}, r_{m}\right) \alpha\left(r_{n+2}, r_{n+3}\right) \rho_{q}\left(r_{n+2}, r_{n+3}\right) \\
& +\mu\left(r_{n+1}, r_{m}\right) \mu\left(r_{n+2}, r_{m}\right) \mu\left(r_{n+3}, r_{m}\right) \rho_{q}\left(r_{n+3}, r_{m}\right) \\
\leq & \alpha\left(r_{n}, r_{n+1}\right) \rho_{q}\left(r_{n}, r_{n+1}\right) \\
& \quad+\sum_{i=n+1}^{m-2}\left(\prod_{j=n+1}^{i} \mu\left(r_{j}, r_{m}\right)\right) \alpha\left(r_{i}, r_{i+1}\right) \rho_{q}\left(r_{i}, r_{i+1}\right)+\prod_{k=n+1}^{m-1} \mu\left(r_{k}, r_{m}\right) \rho_{q}\left(r_{m-1}, r_{m}\right) \\
\leq & \alpha\left(r_{n}, r_{n+1}\right)\left(\frac{t}{1-t}\right)^{n} \rho_{q}\left(r_{0}, r_{1}\right)+\sum_{i=n+1}^{m-1}\left(\prod_{j=0}^{i} \mu\left(r_{j}, r_{m}\right)\right) \alpha\left(r_{i}, r_{i+1}\right)\left(\frac{t}{1-t}\right)^{i} \rho_{q}\left(r_{0}, r_{1}\right) .
\end{aligned}
$$

Hence, we have

$$
\rho_{q}\left(r_{n}, r_{m}\right) \leq \rho_{q}\left(r_{0}, r_{1}\right)\left[\left(\frac{t}{1-t}\right)^{n} \alpha\left(r_{n}, r_{n+1}\right)+S_{m-1}-S_{n}\right] \text {, }
$$

where

$$
S_{n}=\sum_{i=1}^{n}\left(\prod_{j=0}^{i} \mu\left(r_{j}, r_{m}\right)\right) \alpha\left(r_{i}, r_{i+1}\right)\left(\frac{t}{1-t}\right)^{i} .
$$

By using ratio test and inequality (10), it can be proved easily that the infinite series $\sum_{i=1}^{\infty}\left(\prod_{j=0}^{i} \mu\left(r_{j}, r_{m}\right)\right) \alpha\left(r_{i}, r_{i+1}\right)(t /(1-t))^{i} \quad$ is convergent. Letting $n, m \longrightarrow \infty$ in (25), yields 


$$
\lim _{n, m \longrightarrow \infty} \rho_{q}\left(r_{n}, r_{m}\right)=0 .
$$

So, the sequence $\left\{T S\left(r_{n}\right)\right\}$ is a left Cauchy sequence. Since $\left(\mathfrak{I}, \rho_{q}\right)$ is a left $K$-sequentially double controlled complete dislocated quasi-metric type space, there exists $r^{*} \in \mathfrak{I}$ such that $\left\{T S\left(r_{n}\right)\right\} \longrightarrow r^{*} \in \mathfrak{I}$ and

$$
\lim _{n \longrightarrow \infty} \rho_{q}\left(r_{n}, r^{*}\right)=\lim _{n \longrightarrow \infty} \rho_{q}\left(r^{*}, r_{n}\right)=0 .
$$

Since $\left(\mathfrak{I}, \rho_{q}\right)$ is a left $K$-sequentially complete and $G(S)$ is closed subset of $\mathfrak{T}$, so $\left(G(S), \rho_{q}\right)$ is also left $K$-sequentially complete. As $\beta^{*}\left(r_{2 p}, S r_{2 p}\right) \geq 1$ for all $p \in \mathbb{N}$. So, $\left\{r_{2 n}\right\}$ is a subsequence of $\left\{T S\left(r_{n}\right)\right\}$ contained in $G(S)$. By completeness of $\left(G(S), \rho_{q}\right)$ and uniqueness of limit, $\left\{r_{2 n}\right\} \longrightarrow r^{*} \in G(S)$, that is,

$$
\beta^{*}\left(r^{*}, S r^{*}\right) \geq 1 .
$$

Now, we suppose that $\rho_{q}\left(r^{*}, S r^{*}\right) \neq 0$. By Lemma 1 , we have

$$
\rho_{q}\left(r_{2 n}, S r^{*}\right) \leq H_{\rho_{q}}\left(T r_{2 n-1}, S r^{*}\right) .
$$

As $\beta^{*}\left(r^{*}, S r^{*}\right) \geq 1$ and $\beta^{*}\left(S r_{2 n-1}, r_{2 n-1}\right) \geq 1$, by using (9), we have

$$
\rho_{q}\left(r_{2 n}, S r^{*}\right) \leq t\left[\rho_{q}\left(r_{2 n-1}, r_{2 n}\right)+\rho_{q}\left(r^{*}, S r^{*}\right)\right] .
$$

Taking $\lim _{n \longrightarrow \infty}$ on both sides of inequality (31), we obtain

$$
\begin{aligned}
& \left.\lim _{n \longrightarrow \infty} \rho_{q}\left(r_{2 n}, S r^{*}\right) \leq \lim _{n \longrightarrow \infty} t\left[\rho_{q}\left(r_{2 n-1}, r_{2 n}\right)+\rho_{q}\left(r^{*}, S r^{*}\right)\right)\right], \\
& \lim _{n \longrightarrow \infty} \rho_{q}\left(r_{2 n}, S r^{*}\right) \leq t\left(\rho_{q}\left(r^{*}, S r^{*}\right)\right) .
\end{aligned}
$$

Now,

$\rho_{q}\left(r^{*}, S r^{*}\right) \leq \alpha\left(r^{*}, r_{2 n}\right) \rho_{q}\left(r^{*}, r_{2 n}\right)+\mu\left(r_{2 n}, S r^{*}\right) \rho_{q}\left(r_{2 n}, S r^{*}\right)$.

Taking $\lim _{n \longrightarrow \infty}$ of inequality (33) and using inequality (11) and (28), we obtain

$$
\rho_{q}\left(r^{*}, S r^{*}\right) \leq \lim _{n \longrightarrow \infty} \mu\left(r_{2 n}, S r^{*}\right) \rho_{q}\left(r_{2 n}, S r^{*}\right) .
$$

Using inequalities (11) and (32) in inequality (34), we obtain

$$
\rho_{q}\left(r^{*}, S r^{*}\right)<\rho_{q}\left(r^{*}, S r^{*}\right) .
$$

It is a contradiction; therefore,

$$
\rho_{q}\left(r^{*}, S r^{*}\right)=0 .
$$

Now, suppose that $\rho_{q}\left(S r^{*}, r^{*}\right) \neq 0$. By Lemma 1, we have

$$
\rho_{q}\left(S r^{*}, r_{2 n}\right) \leq H_{\rho_{q}}\left(S r^{*}, T r_{2 n-1}\right) .
$$

Now, $\beta^{*}\left(r^{*}, S r^{*}\right) \geq 1$ and $\beta^{*}\left(S r_{2 n-1}, r_{2 n-1}\right) \geq 1$, so by (9), we have

$$
\rho_{q}\left(S r^{*}, r_{2 n}\right) \leq t\left[\rho_{q}\left(r^{*}, S r^{*}\right)+\rho_{q}\left(r_{2 n-1}, r_{2 n}\right)\right] .
$$

Taking $\lim _{n \longrightarrow \infty}$ on both sides of inequality (38), we obtain

$$
\lim _{n \rightarrow \infty} \rho_{q}\left(S r^{*}, r_{2 n}\right) \leq t\left(\rho_{q}\left(r^{*}, S r^{*}\right)\right) .
$$

Now,

$\rho_{q}\left(S r^{*}, r^{*}\right) \leq \alpha\left(S r^{*}, r_{2 n}\right) \rho_{q}\left(S r^{*}, r_{2 n}\right)+\mu\left(r_{2 n}, r^{*}\right) \rho_{q}\left(r_{2 n}, r^{*}\right)$.

Taking $\lim _{n \rightarrow \infty}$ of inequality (40) and using inequalities (11) and (28), we obtain

$$
\rho_{q}\left(S r^{*}, r^{*}\right)<\frac{1}{t} \lim _{n \longrightarrow \infty} \rho_{q}\left(S r^{*}, r_{2 n}\right) .
$$

By using inequality (39) in inequality (41), we obtain

$$
\rho_{q}\left(S r^{*}, r^{*}\right)<\rho_{q}\left(r^{*}, S r^{*}\right)=0 \text {, by. }
$$

It is a contradiction. Hence, $\rho_{q}\left(r^{*}, S r^{*}\right)=\rho_{q}\left(S r^{*}, r^{*}\right)=$ 0 , so $r^{*} \in S r^{*}$. Now,

$$
\rho_{q}\left(r^{*}, r^{*}\right) \leq \alpha\left(r^{*}, r_{2 n}\right) \rho_{q}\left(r^{*}, r_{2 n}\right)+\mu\left(r_{2 n}, r^{*}\right) \rho_{q}\left(r_{2 n}, r^{*}\right) .
$$

This implies $\rho_{q}\left(r^{*}, r^{*}\right)=0$ as $n \longrightarrow \infty$. As $\beta^{*}\left(r^{*}, S r^{*}\right) \geq 1 \quad$ and $\rho_{q}\left(r^{*}, S r^{*}\right)=\rho_{q}\left(S r^{*}, r^{*}\right)=0=$ $\rho_{q}\left(r^{*}, r^{*}\right)$. So, Definition 6 implies

$$
\beta^{*}\left(S r^{*}, r^{*}\right) \geq 1 \text {. }
$$

Now, by Lemma 1, we have

$$
\rho_{q}\left(r_{2 n+1}, \operatorname{Tr}^{*}\right) \leq H_{\rho_{q}}\left(S r_{2 n}, \operatorname{Tr}^{*}\right) .
$$
have

As, $\beta^{*}\left(r_{2 n}, S r_{2 n}\right) \geq 1$ and $\beta^{*}\left(S r^{*}, r^{*}\right) \geq 1$, so by (9), we

$$
\left.\rho_{q}\left(r_{2 n+1}, \operatorname{Tr}^{*}\right) \leq t\left[\rho_{q}\left(r_{2 n}, r_{2 n+1}\right)+\rho_{q}\left(r^{*}, \operatorname{Tr}^{*}\right)\right)\right] .
$$
obtain

Taking $\lim _{n \longrightarrow \infty}$ on both sides of inequality (46), we $\left.\lim _{n \longrightarrow \infty} \rho_{q}\left(r_{2 n+1}, \operatorname{Tr}^{*}\right) \leq \lim _{n \longrightarrow \infty} t\left[\rho_{q}\left(r_{2 n}, r_{2 n+1}\right)+\rho_{q}\left(r^{*}, \operatorname{Tr}^{*}\right)\right)\right]$.

Letting $n \longrightarrow \infty$ and using inequality (27), we have

$$
\lim _{n \longrightarrow \infty} \rho_{q}\left(r_{2 n+1}, \operatorname{Tr}^{*}\right) \leq t\left(\rho_{q}\left(r^{*}, \operatorname{Tr}^{*}\right)\right) .
$$

Since,

$$
\rho_{q}\left(r^{*}, \operatorname{Tr}^{*}\right) \leq \alpha\left(r^{*}, r_{2 n}\right) \rho_{q}\left(r^{*}, r_{2 n}\right)+\mu\left(r_{2 n+1}, \operatorname{Tr}^{*}\right) \rho_{q}\left(r_{2 n+1}, \operatorname{Tr}^{*}\right) \text {, }
$$

by taking $\lim _{n \longrightarrow \infty}$ and using inequality (11) and (46), we obtain

$$
\rho_{q}\left(r^{*}, \operatorname{Tr}^{*}\right)<\frac{1}{t} \lim _{n \longrightarrow \infty}\left(\rho_{q}\left(r_{2 n+1}, \operatorname{Tr}^{*}\right)\right) .
$$

By using inequalities (11) and (48), we obtain

$$
\rho_{q}\left(r^{*}, \operatorname{Tr}^{*}\right)<\rho_{q}\left(r^{*}, \operatorname{Tr}^{*}\right) .
$$


It is a contradiction. Thus, $\rho_{q}\left(r^{*}, T r^{*}\right)=0$. Similar to the arguments above, we obtain

$$
\rho_{q}\left(\operatorname{Tr}^{*}, r^{*}\right)=0
$$

Hence, $r^{*} \in T r^{*}$. Hence, $r^{*}$ is a common fixed point for $S$ and $T$.

Example 2. Let $\mathfrak{\Im}=[0, \infty) \cap \mathbb{Q}^{+}$. Define $\rho_{q}(r, y)=(r+2 y)^{2}$ if $r \neq y$ and $\rho_{q}(r, y)=0$ if $r=y$. Then, $\left(\mathfrak{I}, \rho_{q}\right)$ is a complete double controlled dislocated quasi-metric type space with

$$
\begin{aligned}
& \alpha(r, y)= \begin{cases}2, & \text { if } r, y \geq 1, \\
\frac{r+2}{2}, & \text { otherwise, }\end{cases} \\
& \mu(r, y)= \begin{cases}1, & \text { if } r, y \geq 1, \\
\frac{y+2}{2}, & \text { otherwise. }\end{cases}
\end{aligned}
$$

Let,

$$
\beta(r, y)= \begin{cases}1, & \text { if } r \in A y \in B, \\ \frac{1}{4}, & \text { otherwise. }\end{cases}
$$

Now,

$$
\begin{aligned}
& A=\left\{r: \beta^{*}(r, S r) \geq 1\right\}=\left\{0,1, \frac{1}{64}, \frac{1}{4096}, \ldots\right\}, \\
& B=\left\{y: \beta^{*}(S y, y) \geq 1\right\}=\left\{0, \frac{1}{8}, \frac{1}{512}, \ldots\right\} .
\end{aligned}
$$

Define the mappings $S, T: \mathfrak{I} \longrightarrow P(\mathfrak{I})$ as follows:

$$
\begin{aligned}
& T(y)= \begin{cases}{\left[\frac{y}{8}, \frac{y}{4}\right] \cap \mathbb{Q}^{+},} & \text {for all } y \in\left\{0,1, \frac{1}{8}, \frac{1}{64}, \frac{1}{512}, \frac{1}{4096}, \ldots\right\}, \\
{[y+2,2(y+1)] \cap \mathbb{Q}^{+},} & \text {if otherwise, }\end{cases} \\
& S(y)= \begin{cases}\left\{\frac{1}{8} y\right\}, & \text { for all } y \in\left\{0,1, \frac{1}{8}, \frac{1}{64}, \frac{1}{512}, \frac{1}{4096}, \ldots\right\}, \\
{[y+1, y+3] \cap \mathbb{Q}^{+},} & \text {if otherwise. }\end{cases}
\end{aligned}
$$

The given $\rho_{q}$ is not a controlled dislocated quasi-metric type space for the function $\alpha$. Indeed,

$$
\rho_{q}(1,3)=49>37.5=\alpha(1,0) \rho_{q}(1,0)+\alpha(0,3) \rho_{q}(0,3) .
$$

Now, $\quad \rho_{q}\left(r_{0}, S r_{0}\right)=\rho_{q} \quad(1, S 1)=\rho_{q}(1,(1 / 8))=(1+$ $(2 / 8))^{2}=(5 / 4)^{2}$, we define the sequence $\left\{\operatorname{TS}\left(r_{n}\right)\right\}=\{1,(1 / 8),(1 / 64),(1 / 512),(1 / 4096), \ldots\}$ in $\mathfrak{J}$ generated by $r_{0}=1$.

Note that $\beta^{*}(r, S r) \geq 1, \quad \rho_{q}(r, S r)=\rho_{q}(r, y), \quad$ and $\rho_{q}(S r, r)=\rho_{q}(y, r)$ imply $\beta^{*}(S y, y) \geq 1$. Also, $\beta^{*}(S r, r) \geq 1$, $\rho_{q}(r, \operatorname{Tr})=\rho_{q}(r, y), \quad$ and $\quad \rho_{q}(\operatorname{Tr}, r)=\rho_{q}(y, r)$ imply $\beta^{*}(y, S y) \geq 1$. So, the pair $(S, T)$ is $\beta^{*}$-Alt multivalued mapping on $\left\{T S\left(r_{n}\right)\right\}$.

Now, for all $r, y \in \mathfrak{I} \cap\left\{T S\left(r_{n}\right)\right\}$ with $\beta^{*}(S y, y) \geq 1$, $\beta^{*}(r, S r) \geq 1$, and $t=(2 / 5)$, we observe that all cases are satisfied, that is,

$$
\max \left\{H_{\rho_{q}}(S r, T y), H_{\rho_{q}}(T r, S y)\right\} \leq t\left(\rho_{q}(r, S r)+\rho_{q}(y, T y)\right) \text {. }
$$

Now, $r_{0}=1$, and we have $r_{1}=(1 / 8), r_{2}=(1 / 64)$, $r_{3}=(1 / 512), \ldots$

$$
\sup _{m \geq 1} \lim _{i \rightarrow \infty} \frac{\alpha\left(r_{i+1}, r_{i+2}\right)}{\alpha\left(r_{i}, r_{i+1}\right)} \mu\left(r_{i+1}, r_{m}\right) \approx 0.8<\frac{1-t}{t}=\frac{3}{2} \text {. }
$$

That is, the pair $(S, T)$ is $\beta^{*}$ Kannan-type double controlled contraction. Let $r_{0}=1$, and we have

$$
\begin{aligned}
G(S) & =\left\{r: \beta^{*}(r, S r) \geq 1 r \in\left\{T S\left(r_{n}\right)\right\}\right\} \\
& =\left\{0,1, \frac{1}{64}, \frac{1}{4096}, \ldots\right\} .
\end{aligned}
$$

That is (i) holds. Finally, for every $r \in\left\{T S\left(r_{n}\right)\right\}$, we have

$$
\begin{array}{r}
\lim _{n \longrightarrow \infty} \alpha\left(r, r_{n}\right)<\frac{5}{2}, \\
\lim _{n \rightarrow \infty}\left(r_{n}, r\right)<\frac{5}{2} .
\end{array}
$$

Thus, all the hypothesis of Theorem 2 are satisfied. In fact, 0 is a common fixed point of $S$ and $T$.

Definition 8. Let $\left(\mathfrak{I}, \rho_{q}\right)$ be a complete dislocated quasi $b$ metric type space and $(S, T)$ be a pair of $\beta^{*}$-Alt multivalued mapping. Then, the pair $(S, T)$ is called $\beta^{*}$ Kannan-type $b$-contraction, if for every two consecutive points $r$ and $y$ 
belonging to the range of an iterative sequence $\left\{T S\left(r_{n}\right)\right\}$ with $\beta^{*}(S y, y) \geq 1$ and $\beta^{*}(r, S r) \geq 1$, and we have

$$
\max \left\{H_{p q}(S y, T r), H_{\rho_{q}}(\operatorname{Tr}, S y)\right\} \leq t\left(\rho_{q}(r, T r)+\rho_{q}(y, S y)\right) \text {, }
$$

whenever, $t \in[0,(1 / 2))$ and

$$
b<\frac{1-t}{t}
$$

Theorem 3. Let $\left(\mathfrak{I}, \rho_{q}\right)$ be a left $K$-sequentially complete dislocated quasi b-metric space and a pair $(S, T)$ be a $\beta^{*}$ Kannan type b-contraction. Assume the following.

The set $G(S)=\left\{r: \beta^{*}(r, S r) \geq 1\right\}$ is closed and contained $r_{0}$. Then, $\left.\left\{T S\left(r_{n}\right)\right\}\right\} \longrightarrow r^{*} \in \mathfrak{J}$. Also, if (62) holds for each $r, y \in\left\{r^{*}\right\}$, then $S$ and $T$ have a common fixed point $r^{*}$ in $\mathfrak{J}$ and $\rho_{q}\left(r^{*}, r^{*}\right)=0$.

Remark 2. In Example 2, $\rho_{q}(r, y)=(r+2 y)^{2}$ is a dislocated quasi $b$-metric with $b=2$, but we cannot apply Theorem 3 because the pair $(S, T)$ is not $\beta^{*}$ Kannan type $b$-contraction, indeed $b \nless(1-t) / t=(3 / 2)$.

\section{Conclusion}

The aim of this work is to introduce double controlled dislocated quasi-metric type spaces and related definitions as a generalization of double controlled metric-type spaces. We have removed one and a half restriction out of three restrictions of double controlled metric-type spaces. This new space is a generalization of metric space, quasi-metric space, dislocated metric space, dislocated quasi-metric space, partial metric space, quasi-partial metric space, $b$-metric space, quasi- $b$-metric space, dislocated $b$-metric space, dislocated quasi- $b$-metric space, extended $b$-metric space, dislocated extended $b$-metric space, quasi-extended $b$-metric space, dislocated quasi-extended $b$-metric space, and double controlled metric space. We establish a new generalized contraction on a sequence and obtain fixed point results for a pair of multivalued mappings in left $k$-sequentially complete double controlled dislocated quasi-metric type spaces. New results in ordered spaces and new results for graphic contractions can be obtained as corollaries of our results. An example has been built to demonstrate the novelty of results. [29]

\section{Data Availability}

No data were used to support this study.

\section{Conflicts of Interest}

The authors declare that they have no conflicts of interest.

\section{Authors' Contributions}

Each author equally contributed to this paper and read and approved the final manuscript.

\section{References}

[1] J. P. Aubin, Mathematical Methods of Games and Economic Theory, North-Holland, Amsterdam, Netherlands, 1979.

[2] S. Carl and S. Heikkila, Fixed Point Theory in Ordered Sets and Applications from Differential and Integral Equations to Game Theory, Springer, New York, NY, USA, 2010.

[3] S. Bohnenblust and S. Karlin, Contributions to the Theory of Games, Princeton University Press, Princeton, NJ, USA, 1950.

[4] J. L. Li, "Several extensions of the Abian-Brown fixed point theorem and their applications to extended and generalized Nash equalibria on chain-complete posets," Journal of Mathematical Analysis and Applications, vol. 409, no. 2, pp. 1084-1092, 2017.

[5] H. Aydi, M. F. Bota, E. Karapinar, and S. Mitrović, "Fixed point theorem for set-valued quasi-contractions in b-metric spaces," Fixed Point Theory Applications, vol. 2012, no. 88, p. 8, 2012.

[6] T. Rasham, A. Shoaib, N. Hussain, B. A. S. Alamri, and M. Arshad, "Multivalued fixed point results in dislocated bmetric spaces with application to the system of nonlinear integral equations," Symmetry, vol. 40, no. 11, p. 16, 2019.

[7] T. Rasham, A. Shoaib, B. A. S. Alamri, and M. Arshad, "Multivalued fixed point results for new generalized F-dominated contractive mappings on dislocated metric space with application," Journal of Function Spaces, vol. 2018, Article ID 4808764, 12 pages, 2018.

[8] T. Rasham, A. Shoaib, B. A. S. Alamri, and M. Arshad, "Fixed point results for multivalued contractive mappings endowed with graphic structure," Journal of Mathematics, vol. 2018, Article ID 5816364, 8 pages, 2018.

[9] M. Sgroi and C. Vetro, "Multi-valued F-contractions and the solutions of certain functional and integral equations," Filomat, vol. 27, no. 7, pp. 1259-1268, 2013.

[10] A. Shoaib, T. Rasham, N. Hussain, and M. Arshad, " $\alpha *$-dominated set-valued mappings and some generalised fixed point results," Journal of the National Science Foundation of Sri Lanka, vol. 47, no. 2, pp. 235-243, 2019.

[11] A. Shoaib, "Fixed point results for $\alpha *-\psi$-multivalued mappings," Bulletin of Mathematical Analysis and Applications, vol. 8, no. 4, pp. 43-55, 2016.

[12] A. Shoaib, A. Azam, M. Arshad, and A. Shahzad, "Fixed point results for the multivalued mapping on closed ball in dislocated fuzzy metric space," Journal of Mathematical Analysis, vol. 8, no. 2, pp. 98-106, 2017.

[13] A. Shoaib, A. Azam, and A. Shahzad, "Common fixed point results for the family of multivalued mappings satisfying contraction on a sequence in Hausdorff fuzzy metric space," Journal of Computational Analysis and Applications, vol. 24, no. 4, pp. 692-699, 2018.

[14] A. Shoaib, A. Hussain, M. Arshad, and A. Azam, "Fixed point results for $\alpha *-\psi$-Ciric type multivalued mappings on an intersection of a closed ball and a sequence with graph," Journal of Mathematical Analysis, vol. 7, no. 3, pp. 41-50, 2016.

[15] F. Gu and L. Wang, "Some coupled fixed-point theorems in two quasi-partial metric spaces," Fixed Point Theory Applications, vol. 2014, no. 19, p. 17, 2014.

[16] E. Karapınar, İ.M. Erhan, and A. Öztürk, "Fixed point theorems on quasi-partial metric spaces," Mathematical and Computer Modelling, vol. 57, no. 9-10, pp. 2442-2448, 2013.

[17] N. Mlaiki, H. Aydi, N. Souayah, and T. Abdeljawad, "Controlled metric type spaces and the related contraction principle," Mathematics, vol. 194, no. 6, p. 7, 2018. 
[18] S. S. Alshoraify, A. Shoaib, and M. Arshad, "New types of F-contraction for multivalued mappings and related fixed point results in abstract spaces," Journal of Function Spaces, vol. 2019, Article ID 1812461, 14 pages, 2019.

[19] M. Arshad, Z. Kadelburg, S. Radenovic, A. Shoaib, and S. Shukla, "Fixed points of $\alpha$-dominated mappings on dislocated quasi metric spaces," Filomat, vol. 31, no. 11, pp. 3041-3056, 2017.

[20] M. Sarwar, M. U. Rahman, and G. Ali, "Some fixed point results in dislocated quasi metric (dq-metric) spaces," Journal of Inequalities and Applications, vol. 2014, Article ID 278, 11 pages, 2014.

[21] F. M. Zeyada, G. H. Hassan, and M. A. Ahmed, "A generalization of a fixed point theorem due to Hitzler and Seda in dislocated quasi-metric spaces," Arabian Journal for Science and Engineering, vol. 31, no. 1, pp. 111-114, 2006.

[22] L. Zhu, C. Zhu, C. Chen, and Z. Stojanović, "Multidimensional fixed points for generalized $\Psi$-quasi-contractions in quasi-metric-like spaces," Journal of Inequalities and Applications, vol. 2014, no. 27, p. 15, 2014.

[23] A. C. M. Ran and M. C. B. Reurings, "A fixed point theorem in partially ordered sets and some applications to metrix equations," Proceedings of the American Mathematical Society, vol. 132, no. 05, pp. 1435-1444, 2004.

[24] J. J. Nieto and R. Rodríguez-López, "Contractive mapping theorems in partially ordered sets and applications to ordinary differential equations," Order, vol. 22, no. 3, pp. 223-239, 2005.

[25] I. Altun, N. A. Arifi, M. Jleli, A. Lashin, and B. Samet, “A new approach for the approximations of solutions to a common fixed point problem in metric fixed point theory," Journal of Function Spaces, vol. 2016, Article ID 6759320, 5 pages, 2016.

[26] T. Kamran, M. Samreen, and O. U. A. Ain, "Generalization of b-metric space and some fixed point theorems," Mathematics, vol. 19, no. 5, p. 7, 2017.

[27] M. Samreen, T. Kamran, and M. Postolache, "Extended b-metric space, extended b-comparison function and nonlinear contractions," UPB Scientific Bulletin, Series A: Applied Mathematics and Physics, vol. 80, no. 4, pp. 21-28, 2018.

[28] T. Abdeljawad, N. Mlaiki, H. Aydi, and N. Souayah, "Double controlled metric type spaces and some fixed point results," Mathematics, vol. 320, no. 6, p. 10, 2018.

[29] A. Shoaib, M. Arshad, and M. A. Kutbi, "Common fixed points of a pair of Hardy Rogers type mappings on a closed ball in ordered partial metric spaces," Journal of Computational Analysis and Applications, vol. 17, no. 2, pp. 255-264, 2014. 